\title{
Serum procalcitonin and C-reactive protein concentrations to distinguish mildly infected from non-infected diabetic foot ulcers: a pilot study
}

\author{
A. Jeandrot • J.-L. Richard $\cdot$ C. Combescure • \\ N. Jourdan $\cdot$ S. Finge $\cdot$ M. Rodier $\cdot$ P. Corbeau $•$ \\ A. Sotto - J.-P. Lavigne
}

Received: 30 July 2007 /Accepted: 31 August 2007 / Published online: 13 October 2007

(C) Springer-Verlag 2007

\begin{abstract}
Aims/hypothesis Infection of diabetic foot ulcers is common; at early stages it is difficult to differentiate between non-infected ulcers (or those colonised with normal flora) and ulcers infected with virulent bacteria that lead to deterioration. This pilot study aimed to assess the diagnostic accuracy of inflammatory markers as an aid to making this distinction.
\end{abstract}

\author{
A. Jeandrot $\cdot$ J.-P. Lavigne \\ Department of Bacteriology, \\ University Hospital Caremeau, \\ Nimes, France \\ J.-L. Richard \\ Diabetic Foot Unit, University Hospital Nimes, \\ Le Grau du Roi, France \\ C. Combescure \\ Department of Medical Information, \\ University Hospital Caremeau, \\ Nimes, France \\ N. Jourdan $\cdot$ M. Rodier \\ Department of Endocrinology, \\ University Hospital Caremeau, \\ Nimes, France \\ S. Finge $\cdot$ P. Corbeau \\ Department of Immunology, \\ University Hospital Caremeau, \\ Nimes, France
}

A. Sotto $·$ J.-P. Lavigne $(\square)$
Institut National de la Santé et de
ESPRI 26, Université Montpellier
Avenue Kennedy,
30908 Nimes Cedex 02, France
e-mail: jphlavigne@hotmail.com
Methods We included 93 diabetic patients who had an episode of foot ulcer and had not received antibiotics during the 6 months preceding the study. Ulcers were classified as infected or uninfected, according to the Infectious Diseases Society of America-International Working Group on the Diabetic Foot classification. Diabetic patients without ulcers $(n=102)$ served as controls. C-reactive protein (CRP), orosomucoid, haptoglobin and procalcitonin were measured together with white blood cell and neutrophil counts. The diagnostic performance of each marker, in combination (using logistic regression) or alone, was assessed.

Results As a single marker, CRP was the most informative for differentiating grade 1 from grade 2 ulcers (sensitivity 0.727 , specificity 1.000 , positive predictive value 1.000 , negative predictive value 0.793 ) with an optimal cut-off value of $17 \mathrm{mg} / \mathrm{l}$. In contrast, white blood cell and neutrophil counts were not predictive. The most relevant combination derived from the logistic regression was the association of CRP and procalcitonin (AUC 0.947), which resulted in a significantly more effective determination of ulcer grades, as shown by comparing receiver operating characteristic curves.

Conclusions/interpretation Measurement of only two inflammatory markers, CRP and procalcitonin, might be of value for distinguishing between infected and non-infected foot ulcers in subgroups of diabetic patients, to help ensure the appropriate allocation of antibiotic treatment. Nevertheless, external validation of the diagnostic value of procalcitonin and CRP in diabetic foot ulcers is needed before routine use can be recommended.

Keywords C-reactive protein - Diabetic foot ulcer . Diagnostic · Infection - Inflammatory markers · IWGDF-IDSA classification · Procalcitonin 


$\begin{array}{ll}\text { Abbreviations } \\ \text { CRP } & \text { c-reactive protein } \\ \text { IDSA } & \text { Infectious Diseases Society of America } \\ \text { IWGDF } & \text { International Working Group on the Diabetic } \\ & \begin{array}{l}\text { Foot } \\ \text { receiver operating characteristic }\end{array}\end{array}$

\section{Introduction}

Foot ulcers are common in diabetic patients, with 15 to $25 \%$ estimated to experience such an ulcer during their lifetime [1]. These ulcers frequently become infected and the spread of infections to soft tissue and eventually to bone structures is a major causal factor for lower-limb amputation [2-5]. Early diagnosis and adequate treatment are therefore essential to prevent amputation. As all skin wounds harbour microorganisms, some physicians advocate a prophylactic antibiotic therapy for treatment of clinically uninfected ulcers in order to eradicate potential pathogens [6]. This, however, kills commensal flora and may impair healing as well as possibly leading to a real infection. However, as emphasised by the Infectious Diseases Society of America (IDSA), available evidence does not support such a concept [7]. In 2004, both the IDSA and the International Working Group on the Diabetic Foot (IWGDF) published a system for grading infection severity $[7,8]$, the clinical benefit of which was recently established [9]. Both working groups emphasised that diagnosis of infection must be based not on microbiological findings but on clinical criteria. Failure to follow these recommendations results in unnecessary antimicrobial treatment, leading to emergence of multidrug-resistant bacterial strains, increasing costs and possibly causing drug-related adverse events [7]. It is therefore of paramount importance to assess strategies for differentiating non-infected from infected wounds at an early stage.

Biochemical parameters such as sedimentation rate, leucocytosis and circulating inflammatory proteins are reputed to be of poor value for diagnosing diabetic foot infections, as even in the most severe cases there are few systemic manifestations [10, 11]. Procalcitonin, a 116 amino acid protein precursor of the hormone calcitonin, has recently gained acceptance as a marker for diagnosing infection [12-15]. Some authors claim that its accuracy as a predictor of bacterial infection is higher than that of C-reactive protein (CRP) [12].

The aim of the present pilot study was to evaluate the usual biological inflammatory parameters (notably CRP and procalcitonin) to determine if any are able to differentiate between infected and non-infected diabetic foot ulcers.

\section{Methods}

Study design Figure 1 gives an overview of the study design. The study was approved by the ethics committee of Caremeau University Hospital and was carried out in accordance with the Declaration of Helsinki as revised in 2000. Between 1 October 2005 and 31 December 2006, we prospectively recruited all diabetic patients hospitalised for diabetic foot ulcers in our specialised diabetic foot department at the University Hospital in Nimes. Informed consent was obtained. Patients were included if they had received no antimicrobial treatment in the preceding 6 months. Every patient was examined by a trained physician in our department (J.-L. Richard, N. Jourdan) to grade infection severity according to the IDSA-IWGDF criteria (summarised in the Text box: IDSA-IWGDF clinical classification of diabetic foot infection [8]). All wounds were evaluated for any evidence of osteomyelitis by clinical examination, probe-to-bone test [16] and, if necessary, by imaging procedures. At the end of the examination, wounds were considered either non-infected (grade 1) or infected (grade 22 ). Only clinically infected wounds were treated with antibiotics. Patients were followed up for 1 month by the same trained physicians to definitively establish the wound status (infected vs non-infected) using the IDSA-IWGDF clinical criteria. In the event of secondary occurrence of clinical infection, the wound was definitively classified as infected for statistical analysis.

Control participants $(n=102)$ were diabetic patients who had been admitted to hospital for diabetes-related medical problems, but were free from foot wounds and known systemic illnesses that might cause elevation of inflammatory markers. Control participants were matched for age, sex, type of diabetes and complications of diabetes, after informed consent was obtained. None of the control persons was currently being treated with antibiotics.

Biological parameters At inclusion in the study, white blood cell and neutrophil counts, as well as serum concentration of orosomucoid, haptoglobin, albumin, CRP and procalcitonin were determined from a venous sample withdrawn before eventual initiation of antimicrobial treatment (for grade $\geq 2$ ). Blood samples were centrifuged $(4,500 \mathrm{~g})$ within $30 \mathrm{~min}$ of collection. Orosomucoid, haptoglobin, albumin and CRP levels were measured by rate immuno-nephelometry method using an analyser (BN II; Dade Behring, Marburg, Germany). For CRP levels lower than $6 \mathrm{mg} / \mathrm{l}$, the high-sensitivity CRP assay was used. For procalcitonin, $0.2 \mathrm{ml}$ of venous plasma was stored at $-80^{\circ} \mathrm{C}$ for later analysis as a batch to minimise variance. The time-resolved amplified cryptate emission (TRACE) method was used on a Kryptor analyser (BRAHMS Diagnostica, Berlin, Germany). The sensitivity of the functional assay is reported to be $0.04 \mathrm{ng} / \mathrm{ml}$ with intra- 


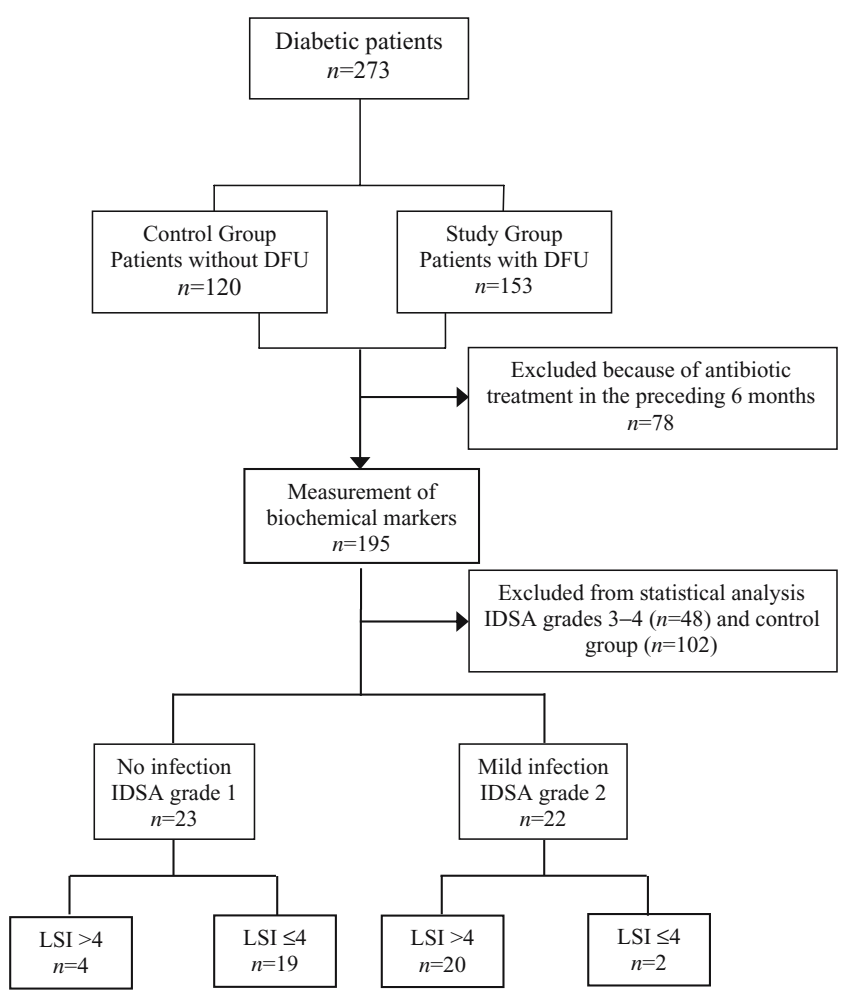

Fig. 1 Flow diagram of the study. DFU diabetic foot ulcers, $L S I$ likelihood score for infection

assay and inter-assay coefficients of variation lower than 5\% and $10 \%$, respectively [17]. White blood cell and differential counts were performed on a haematology analyser (Coulter GEN-S; Beckman Coulter, Fullerton, CA, USA). To minimise bias, laboratory technicians were kept blind to the clinical data and clinicians were unaware of the biochemical results until they had decided whether to initiate antibiotic treatment.

Statistical analysis For a pilot study, an AUC for the receiver operating characteristic (ROC) curve $\left(\mathrm{AUC}_{\mathrm{ROC}}\right)$ of 0.9 , with a precision of 0.1 , was considered as adequate, requiring inclusion of 40 patients with diabetic foot ulcers according to Zhou's formula [18], with patients divided roughly equally into grade 1 and 2 .

Biochemical parameters are given as the median and the range, and compared using a Kruskal-Wallis test. As the aim of our study was to assess value of inflammatory markers in distinguishing grade 1 from grade 2 ulcers, we focused the statistical analyses on these two grades. Data for other grades are given for information. For each single biological marker, an ROC curve was drawn and the $\mathrm{AUC}_{\mathrm{ROC}}$ calculated by the non-parametric Hanley's method [19]. To assess the use of combining several biological markers, we used a logistic regression with a backward procedure to select the most relevant markers; only markers for which $\mathrm{AUC}_{\mathrm{ROC}}$ was greater than 0.80 were initially entered as explanatory variables in the regression analysis. A ROC curve was then generated for the combination derived from the regression model and its area compared with that of every single biological marker by a non-parametric method adapted to paired data [20]. For each ROC curve (single or combined markers), the optimal cut-off value was determined by minimising the number of misclassified ulcers (number of false positives + number of false negatives) and sensitivity, specificity and predictive (negative and positive) value were calculated. Statistical tests results were considered significant at $p<0.05$. Statistical analysis was performed using a software package (S-Plus 2000; Insightful Corporation, Seattle, WA, USA).

\section{Results}

Patient and foot characteristics General patient characteristics were similar in the two studied populations (Table 1). Of 153 patients with diabetic foot ulcers, 93 were included in the study, whereas 60 were excluded because they had received antibiotics in the preceding 6 months. Twentythree wounds $(24.7 \%)$ were classified as grade 1, 22 ulcers as grade $2(23.7 \%), 41$ as grade $3(44.1 \%)$ and seven as grade $4(7.5 \%)$.

IDSA-IWGDF clinical classification of diabetic foot infection [8]

Clinical manifestations of infection

$\begin{array}{ll}\text { Infection severity } & \text { Grade } \\ \text { Uninfected } & 1 \\ \text { Mild } & 2 \\ & \\ \text { Moderate } & 3 \\ & \\ \text { Severe } & 4\end{array}$

Wound lacking purulence or any manifestations of inflammation

Presence of $\geq 2$ manifestations of inflammation (purulence or erythema, pain, tenderness, warmth or induration),

but any cellulitis/erythema extends $\leq 2 \mathrm{~cm}$ around the ulcer and infection is limited to skin or superficial

subcutaneous tissues; no other local complications of systemic illness

Infection (as above) in a patient who is systematically well and metabolically stable, but with $\geq 1$ of the following

characteristics: cellulitis extending $>2 \mathrm{~cm}$, lymphangitic streaking, spread beneath the superficial fascia, deep-tissue

abscess, gangrene, involvement of muscle, tendon, joint or bone

Infection in a patient with systemic toxicity or metabolic instability, e.g. fever, chills, tachycardia, hypotension,

confusion, vomiting, leucocytosis, acidosis, severe hyperglycaemia or azotaemia 
Table 1 General characteristics of the patients

\begin{tabular}{lll}
\hline & $\begin{array}{l}\text { Patients with foot } \\
\text { ulcers }(n=93)\end{array}$ & $\begin{array}{l}\text { Control population } \\
(n=102)\end{array}$ \\
\hline $\begin{array}{l}\text { Age, years } \\
\text { Men }\end{array}$ & $\begin{array}{l}68(43-95) \\
\begin{array}{l}\text { Diabetes mellitus } \\
\text { type 2/type 1 }\end{array}\end{array}$ & $\begin{array}{l}64(26-90) \\
61(59.8)\end{array}$ \\
$\begin{array}{l}\text { HbA } 1 \text { c }(\%) \\
\begin{array}{l}\text { Duration of } \\
\text { diabetes, years }\end{array}\end{array}$ & $7.5(4.9-9.3)$ & $85(83.3) / 17(16.7)$ \\
$\begin{array}{l}\text { Cardiovascular } \\
\text { disease }\end{array}$ & $56(60.2)$ & $7.3(4.2-9.4)$ \\
$\begin{array}{l}\text { Peripheral vascular } \\
\text { disease }\end{array}$ & $42(45.2)$ & $17.3(1-33)$ \\
$\begin{array}{l}\text { Neuropathy } \\
\text { Nephropathy }\end{array}$ & $80(86.0)$ & $58(56.9)$ \\
$\begin{array}{l}\text { Diabetic } \\
\text { retinopathy }\end{array}$ & $59(63.4)$ & $37(35.9)$ \\
\hline
\end{tabular}

Values are given as mean (range) or $n(\%)$

There were no statistically significant differences $(p>0.05)$ between the group of patients with foot ulcers and the controls for any parameter

Baseline results Comparisons between groups are shown on Table 2. No significant difference between patients with grade 1 ulcers and control persons was found for each marker. All markers were significantly higher in grade 2 ulcers than in participants with grade 1 ulcers and in control persons, with the exception of white blood cells. Of note are the twofold increase in procalcitonin and the fourfold increase in CRP level in grade 2 ulcer patients compared with grade 1 .

Statistical analysis $\mathrm{AUC}_{\mathrm{ROC}}$ for distinguishing grade 1 from grade 2 ulcers was greater than 0.800 for all of the biochemical inflammatory markers except albumin (Table 2). On the other hand, $\mathrm{AUC}_{\mathrm{ROC}}$ for haematological parameters was consistently low. According to $\mathrm{AUC}_{\mathrm{ROC}}, \mathrm{CRP}$ was the most informative single parameter (Fig. 2): for a cut-off value

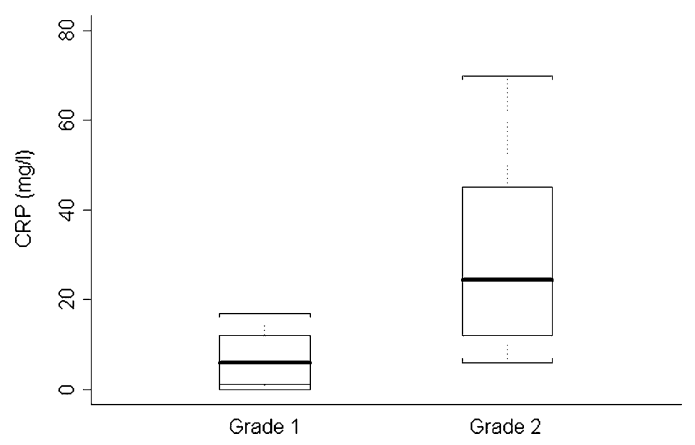

Fig. 2 Distribution of CRP levels in the patients with grade 1 and grade 2 ulcers. Thick lines, median; rectangle, interval between the $1 \mathrm{st}$ and 3rd quartiles; dotted lines and error bars, 95\% CI

of $17 \mathrm{mg} / \mathrm{l}$, sensitivity was 0.727 (SD 0.099 ), specificity 1.000 (SD 0.043), positive predictive value 1.000 (SD 0.052) and negative predictive value 0.793 (SD 0.079).

From the logistic model with backward procedure, combining CRP and procalcitonin provided the most relevant formula for distinguishing between grade 1 and grade 2 ulcers. $\mathrm{AUC}_{\mathrm{ROC}}$ for combination of CRP and procalcitonin $(0.947 \pm$ $0.029)$ was significantly greater than that of CRP or procalcitonin alone $(p<0.05)$ (Fig. 3). The resulting equation (likelihood score for infection) for combining these two parameters was $(0.162 \times \mathrm{CRP} \mathrm{mg} / \mathrm{l})+(17.437 \times$ procalcitonin $\mathrm{ng} / \mathrm{l})$. For an optimal cut-off value of 4 , sensitivity was 0.909 (SD 0.061 ), specificity 0.826 (SD 0.079 ), positive predictive value 0.833 (SD 0.079), negative predictive value 0.905 (SD 0.064), and false-positive $(n=4)$ and false negative $(n=2)$ rates 8.9 and $4.4 \%$, respectively. The Youden index was 0.73 and the positive likelihood ratio 5.2.

Using the formula, six patients were found to have been misclassified: at follow-up, the four grade 1 ulcers with a score greater than 4 healed without antibiotic treatment and the two grade 2 ulcers whose score was lower than 4 healed with appropriate antibiotic treatment. Interestingly, no ulcer classified as grade 1 deteriorated as a result of not being treated - all were reported to have healed.

Table 2 Circulating levels of biochemical markers in the studied population

\begin{tabular}{lllllll}
\hline & Control $(n=102)$ & Grade 1 $(n=23)$ & Grade 2 $(n=22)$ & Grade 3 $(n=41)$ & Grade 4 $(n=7)^{\text {AUC }_{\text {ROC }}{ }^{\mathrm{a}}}$ \\
\hline Orosomucoid $(\mathrm{g} / \mathrm{l})$ & $0.85(0.4-1.32)$ & $0.92(0.5-2.10)$ & $1.35^{\mathrm{b}, \mathrm{c}}(0.6-2.10)$ & $1.36(0.5-3.0)$ & $1.60(1.3-2.40)$ & $0.838(0.063)$ \\
Haptoglobin $(\mathrm{g} / \mathrm{l})$ & $1.71(0.4-3.23)$ & $1.55(0.3-3.90)$ & $2.78^{\mathrm{b}, \mathrm{c}}(1.2-4.20)$ & $2.71(1.3-4.7)$ & $3.22(2.3-5.0)$ & $0.813(0.065)$ \\
Albumin $(\mathrm{g} / \mathrm{l})$ & $37.3(35.2-53.7)$ & $39.6(32.5-46.9)$ & $35.6^{\mathrm{b}, \mathrm{c}}(24.3-47.4)$ & $34.0(18.0-46.0)$ & $30.3(23.4-40.3)$ & $0.695(0.081)$ \\
CRP $(\mathrm{mg} / \mathrm{l})$ & $5.45(0.16-18)$ & $6.00(0.2-19)$ & $24.50^{\mathrm{b}, \mathrm{c}}(6.0-199)$ & $34.00(1.2-357)$ & $146.00(87-255)$ & $0.893(0.045)$ \\
Procalcitonin $(\mathrm{ng} / \mathrm{ml})$ & $0.04(0.0-0.1)$ & $0.07(0.0-0.2)$ & $0.20^{\mathrm{b}, \mathrm{c}}(0.1-0.9)$ & $0.20(0.1-1.4)$ & $0.84(0.5-21.1)$ & $0.881(0.049)$ \\
White blood cells $\left(\times 10^{9} / 1\right)$ & $6.7(4.5-10.1)$ & $6.9(4.4-11.1)$ & $8.0(3.5-12.8)$ & $7.6(4.7-25.5)$ & $15.3(12.1-18.5)$ & $0.661(0.083)$ \\
Neutrophils $\left(\times 10^{9} / \mathrm{l}\right)$ & $4.2(17.6-7.2)$ & $4.0(2.2-9.4)$ & $5.2^{\mathrm{b} . \mathrm{c}}(2.3-10.2)$ & $4.9(2.8-23.9)$ & $13.2(7.9-16.5)$ & $0.700(0.079)$ \\
\hline
\end{tabular}

Values are given as median (range), unless otherwise stated

${ }^{a}$ AUC of the corresponding receiver operating characteristic curve (SD)

${ }^{\mathrm{b}} p<0.05$ vs control

${ }^{\mathrm{c}} p<0.05$ vs grade 1 


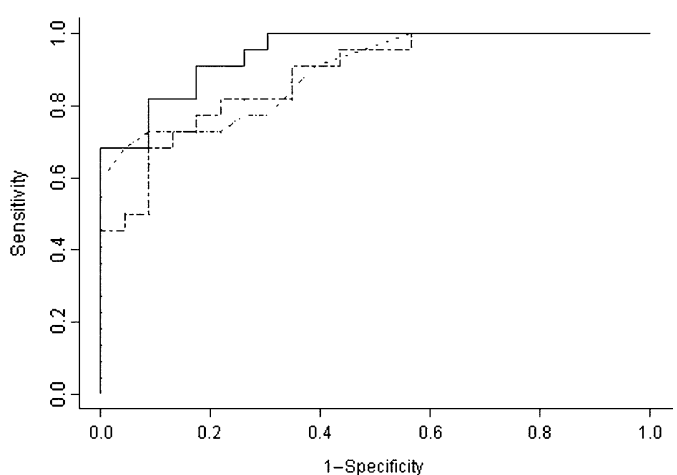

Fig. 3 ROC curves using CRP (dashed and dotted line) and procalcitonin (dashed line) alone or in combination (solid line) $(0.161[\mathrm{CRP}]+17.437$ [procalcitonin] $)$

\section{Discussion}

To our knowledge, our study is the first aiming to determine the value of CRP, procalcitonin and other usual biological inflammatory markers in differentiating infected from noninfected ulcers in early stages of diabetic foot ulcer. Indeed, this study showed that CRP as a single marker had the highest sensitivity and specificity. It was interesting to note that the use of a high-sensitivity CRP assay brought no additional accuracy of diagnosis. Moreover, these blood test results and the follow-up of patients (particularly grade 1 ulcers) could help validate the clinical grading system proposed by the IDSA-IWGDF. Our study suggests that addition of two markers might increase the clinical value of this classification, notably for distinguishing grade 1 from grade 2 ulcers, resulting in better management of diabetic foot ulcers.

The higher performance of CRP in detecting infection, compared with procalcitonin, may be explained by the mild nature of infection in grade 2 diabetic foot ulcers. Indeed, procalcitonin is not a marker of infection as such, since local infection without systemic manifestations results only in a limited increase in procalcitonin levels [21]. On the other hand, CRP values have been shown to significantly increase in response to local infection [21]. $\mathrm{AUC}_{\mathrm{ROC}}$ for orosomucoid and haptoglobin was $>0.8$, but their accuracy was lower than that of both procalcitonin and CRP (data not shown).

In presence of grade 1 ulcers, the values of the biological markers we measured remained normal. Moreover, white blood cells and neutrophil counts were shown to be of little value in diagnosing mild infection in diabetic foot ulcers, as there was no significant difference between grade 2 and grade 1 ulcer patients or the control group: this poor informative potential of haematological parameters confirms the findings of previous studies [10, 11].

The main finding of this prospective study was that combining measurements of CRP and procalcitonin increased the accuracy of predicting wound infection. The simple formula we developed might help clinicians in everyday practice, contributing to earlier diagnosis and possibly reducing exposure to unnecessary antibiotics. Interestingly, the follow-up of patients indicated that this formula was predictive of wound outcome in grade 2 ulcers, since ulcers in all patients with a low score $(<4)$ healed faster than the other ulcers. This observation suggests that a false negative score in the grade 2 ulcer classification would be of little consequence for the patient.

From an immunological point of view, increased CRP and probably procalcitonin concentrations are a hallmark of TNF- $\alpha$-, IL-6- and IL-1-mediated immune response. It means that the presence of bacteria was rated as a threat by the immune system, inducing as a consequence a biological response, which is a fair indication for the clinician.

This study has several limitations. First, diagnosis of infection was based only on clinical examination and many of the characteristic signs of inflammation (i.e. pain, redness, swelling or warmth) are difficult to assess objectively. Moreover, these signs are often diminished in diabetic foot ulcers because of the presence of neuropathy and/or peripheral arterial disease [22]. Second, no attempt to quantify bacterial load was made. Indeed, measurement of bacterial burden has been proposed as an objective means to diagnose wound infection, with a critical level of bacteria $\geq 10^{4}$ to $10^{6}$ colonyforming units per $\mathrm{g}$ of tissue [23]. Using this criterion, it was suggested that clinical assessment of chronic wound infection is unreliable [24]. Moreover, recently an inverse relationship between wound healing rate and bacterial load in wound fluid was proposed to identify diabetic patients with foot ulcers requiring antibiotherapy in borderline cases [25]. Quantitative biopsy is time- and cost-consuming, requires a specialised technician, is not standardised and causes an (unnecessary) trauma [26, 27]. Moreover, the use of measuring bacterial load is further compromised by polymicrobial flora in diabetic foot ulcers and variable virulence of the different microorganisms $[27,28]$. Finally, as we excluded patients with systemic diseases that might increase serum inflammatory markers, it is likely that the performance of CRP and procalcitonin in predicting wound infection would be lower under routine care conditions than in the present analysis. Therefore, the results of our pilot study need validation through further studies in different settings by other investigators (external validation), before randomised controlled trials are carried out to assess the efficacy and cost-effectiveness of a treatment based on the results of serum CRP and procalcitonin measurement.

In conclusion, the measurement of both CRP and procalcitonin may be an interesting tool in diabetic foot ulcer management. Assays for determining CRP and procalcitonin are relatively easy to perform and cause low-to-moderate cost (CRP US\$5, procalcitonin US\$10) with rapidly available results. Combining procalcitonin and CRP values, together with the use of a simple diagram, may allow the early 
distinction between grade 1 and 2 diabetic foot ulcers, so that antibiotic treatment is prescribed to those most likely to receive a benefit.

Acknowledgements The authors are very grateful to D. O'Callaghan for his assistance with this manuscript. J.-P. Lavigne has received grants from the Fondation Coloplast (Coloplast Foundation) and the Association de Langue Française pour l'Etude du Diabète et des Maladies Métaboliques (the French-Speaking Diabetes Association) (ALFEDIAM-Aventis grant). A. Sotto has received grants from the Fondation pour la Recherche Médicale Languedoc-RoussillonRouergue (Languedoc-Roussillon-Rouergue Foundation for Medical Research). INSERM Espri 26 is supported by INSERM, Université de Montpellier 1, the town of Nimes and the region of Languedoc Roussillon.

Duality of interest The authors declare that there is no duality of interest associated with this manuscript.

\section{References}

1. Cavanagh PR, Lipsky BA, Bradbury AW, Botek G (2005) Treatment for diabetic foot ulcers. Lancet 366:1725-1735

2. Mayfield JA, Reiber GE, Sanders LJ, Janisse D, Pogach LM (1998) Preventive foot care in people with diabetes. Diabetes Care 21:2161-2177

3. Boulton AJ, Vileikyte L, Ragnarson-Tennvall G, Apelqvist J (2005) The global burden of diabetic foot disease. Lancet 336:1719-1724

4. Lavery LA, Armstrong DG, Wunderlich RP, Mohler MJ, Wendel CS, Lipsky BA (2006) Risk factors for foot infections in individuals with diabetes. Diabetes Care 29:1288-1293

5. Frykberg RG, Zgonis T, Armstrong DG et al (2006) Diabetic foot disorders: a clinical practice guideline (2006 revision). J Foot Ankle Surg 45:S1-S66

6. Edmonds M, Foster A (2004) The use of antibiotics in the diabetic foot. Am J Surg 187:25S-28S

7. Lipsky BA, Berendt AR, Deery HG et al (2004) Diagnosis and treatment of diabetic foot infections. Clin Infect Dis 39:885-910

8. Lipsky BA (2004) A report from the international consensus on diagnosing and treating the infected diabetic foot. Diabetes Metab Res Rev 20:S68-S77

9. Lavery LA, Armstrong DG, Murdoch DP, Peters EJG, Lipsky BA (2007) Validation of the Infectious Diseases Society of America's diabetic foot infection classification system. Clin Infect Dis 44:562-567

10. Armstrong DG, Perales TA, Murff RT, Edelson GW, Welchon JG (1996) Value of white blood cell count with differential in the acute diabetic foot infection. J Am Podiatr Med Assoc 86:224-227.

11. Eneroth M, Apelqvist J, Stenstrom A (1997) Clinical characteristics and outcome in 223 diabetic patients with deep foot infections. Foot Ankle Int 18:716-722
12. Simon L, Gauvin F, Amre DK, Saint-Louis P, Lacroix J (2004) Serum procalcitonin and C-reactive protein levels as markers of bacterial infection: a systematic review and meta-analysis. Clin Infect Dis 39:206-217

13. Assicot M, Gendrel D, Carsin H, Raymond J, Guilbaud J, Bohuon C (1993) High serum procalcitonin concentrations in patients with sepsis and infection. Lancet 341:515-518

14. Hammer S, Meisner F, Dirschedl P, Hammer C (1998) Procalcitonin: a new marker for diagnosis of acute rejection and bacterial infection in patients after heart and lung transplantation. Transpl Immunol 6:235-241

15. Kordek A, Podraza W, Czajka R (2006) Reliability of semiquantitative determination of procalcitonin serum concentrations in neonates. Diagn Microbiol Infect Dis 56:31-34

16. Lavery LA, Armstrong DG, Peters EJ, Lipsky BA (2007) Probe-tobone test for diagnosing diabetic foot osteomyelitis: reliable or relic? Diabetes Care 30:270-274

17. Steinbach G, Rau B, Debard AL et al (2004) Multicenter evaluation of a new immunoassay for procalcitonin measurement on the Kryptor system. Clin Chem Lab Med 42:440-449

18. Zhou XH, Obuchowski NA, McClish DK (2002) Statistical methods in diagnostic medicine, Wiley \& Sons, New York, pp 198-200

19. Hanley JA, McNeil BJ (1982) The meaning and use of the area under a receiver operating characteristic (ROC) curve. Radiology 143:29-36

20. DeLong ER, DeLong DM, Clarke-Pearson DL (1988) Comparing the areas under two or more correlated receiver operating characteristic curves: a nonparametric approach. Biometrics 44:837-845

21. Rothenburger M, Markewitz A, Lenz T et al (1999) Detection of acute phase response and infection: the role of procalcitonin. Clin Chem Lab Med 37:275-279

22. Edmonds M (2005) Infection in the neuroischemic foot. International Journal of Lower Extremity Wounds 4:145-153

23. Bowler PG, Duerden BI, Armstrong DG (2001) Wound microbiology and associated approaches to wound management. Clin Microbiol Rev 14:244-269

24. Serena T, Robson MC, Cooper DM, Ignatius J, on behalf of the Human Genome Sciences Clinical Trial Group (2006) Lack of reliability of clinical/visual assessment of chronic wound infection: the incidence of biopsy-proven infection in venous leg ulcers. Wounds 18:197-202

25. Xu L, McLennan SV, Lo L et al (2007) Bacterial load predicts healing rate in neuropathic diabetic foot ulcers. Diabetes Care 30:378-380

26. Lipsky BA, Berendt AR, Embil J, de Lalla F (2004) Diagnosing and treating diabetic foot infections. Diabetes Metab Res Rev 20: S56-S64

27. Dow G, Browne A, Sibald RG (1999) Infection in chronic wounds: controversies in diagnosis and treatment. Ostomy Wound Manage 45:23-40

28. Kerstein MD (1996) Wound infection: assessment and management. Wounds 8:141-144 